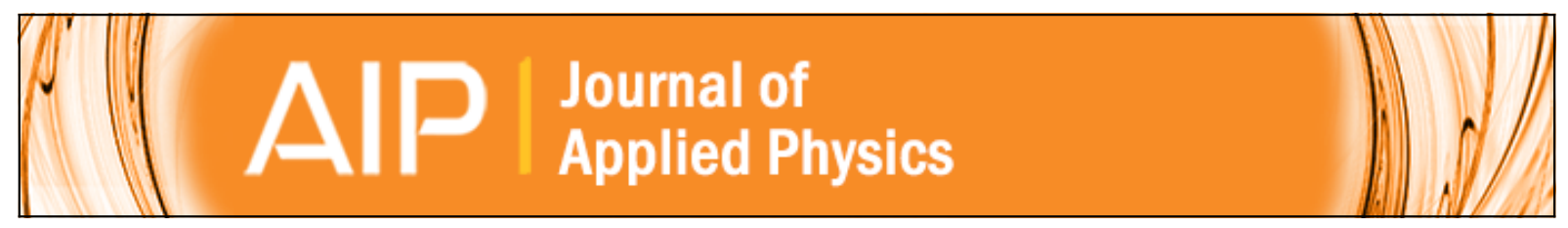

\title{
Magnetoelectric effect in sputtered composites
}

Simon Stein, Manfred Wuttig, Dwight Viehland, and Eckhard Quandt

Citation: Journal of Applied Physics 97, 10Q301 (2005); doi: 10.1063/1.1846631

View online: http://dx.doi.org/10.1063/1.1846631

View Table of Contents: http://scitation.aip.org/content/aip/journal/jap/97/10?ver=pdfcov

Published by the AIP Publishing

\section{Articles you may be interested in}

Converse magnetoelectric effect dependence with CoFeB composition in ferromagnetic/piezoelectric composites J. Appl. Phys. 111, 07 C725 (2012); 10.1063/1.3679443

Magnetoelectric effect in AIN/CoFe bi-layer thin film composites

J. Appl. Phys. 111, 07 C720 (2012); 10.1063/1.3679042

Piezoelectric single crystal langatate and ferromagnetic composites: Studies on low-frequency and resonance magnetoelectric effects

Appl. Phys. Lett. 100, 052901 (2012); 10.1063/1.3679661

Magnetoelectric gyration effect in $\mathrm{Tb} 1-\mathrm{x}$ Dy $x \mathrm{Fe} 2-\mathrm{y} / \mathrm{Pb}(\mathrm{Zr}, \mathrm{Ti}) \mathrm{O} 3$ laminated composites at the electromechanical resonance

Appl. Phys. Lett. 89, 243512 (2006); 10.1063/1.2404977

Magnetoelectric effect in Terfenol- $\mathrm{D} / \mathrm{Pb}(\mathrm{Zr}, \mathrm{Ti} \mathrm{O}) 3 / \mu$-metal laminate composites

Appl. Phys. Lett. 89, 122903 (2006); 10.1063/1.2355459

MIT LINCOLN

LABORATORY

CAREERS

Discover the satisfaction of innovation and service

to the nation
- Space Control

- Air \& Missile Defense

- Communications Systems \& Cyber Security

- Intelligence, Surveillance and

Reconnaissance Systems
- Advanced

Electronics

- Tactical Systems

- Homeland

Protection

- Air Traffic Control

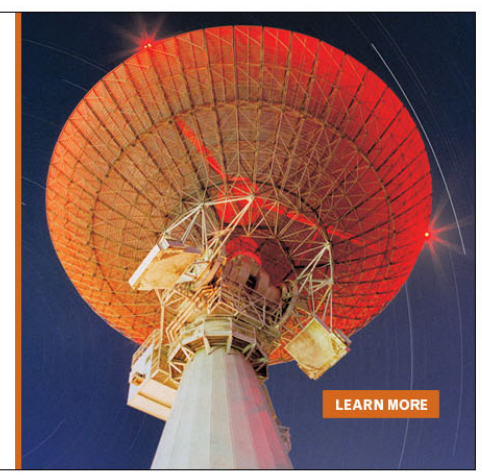




\title{
Magnetoelectric effect in sputtered composites
}

\author{
Simon Stein ${ }^{\text {a) }}$ \\ Smart Materials, Caesar Research Center, 53175 Bonn, Germany \\ Manfred Wuttig \\ Smart Materials, Caesar Research Center, 53175 Bonn, Germany and Department of Materials Science \\ and Engineering, University of Maryland, College Park, Maryland 20742 \\ Dwight Viehland \\ Department of Materials Science and Engineering, Virginia Tech, Blacksburg, Virginia 24061
}

Eckhard Quandt

Smart Materials, Caesar Research Center, 53175 Bonn, Germany

(Presented on 10 November 2004; published online 17 May 2005)

\begin{abstract}
The magnetoelectric effect in millimeter size PMN-PT/Terfenol-D composites is known. In an effort towards miniaturization, we report on the magnetoelectric effect in micrometer-size sputtered composites. Multilayers of $\mathrm{TbFe} / \mathrm{FeCo}$ with a thickness of $4 \mu \mathrm{m}$ were sputter deposited on both sides of PMN-PT piezoelectric single crystals. The magnetoelectric voltage of samples was measured and reached values of $13 \mathrm{mV} /(\mathrm{Oe} \mathrm{cm})$ at dc bias fields of $2 \mathrm{mT}$, a linear dependence of magnetoelectric voltage on ac amplitude was detected in the range from $1 \mathrm{mT}$ to $1 \mathrm{nT}$. (C) 2005 American Institute of Physics. [DOI: 10.1063/1.1846631]
\end{abstract}

\section{INTRODUCTION}

There are natural and composite magnetoelectric (ME) solids. Representatives of the former are $\mathrm{BiFeO}_{3}$ (Refs. 1-4) and $\mathrm{YMnO}_{3}{ }^{5}$ Studies on ME bulk composites originated in The Netherlands in the 1970s and were based on the idea of mechanically coupling highly strictive ferromagnetic and ferroelectric solids. The coefficients relating the magnetic field $H$ and either the electrical polarization $P$ or the electrical field $E$ through the $\mathrm{ME}_{H}$ charge coefficient $\alpha^{p}$ or $\mathrm{ME}_{H}$ voltage coefficient $\alpha^{E}$ would then be given by ${ }^{6}$

$$
\alpha^{P}=\frac{\partial \sigma}{\partial H} \frac{\partial P}{\partial \sigma}
$$

and

$$
\alpha^{E}=\frac{\partial \varepsilon}{\partial H} \frac{\partial E}{\partial \varepsilon} .
$$

It was also shown that $\alpha^{E}$ can be as large as 0.13 $\mathrm{V} /(\mathrm{cm} \mathrm{Oe})$ in the eutectic composites $\mathrm{BaTiO}_{3} /$ $\mathrm{CoFe}_{2} \mathrm{O}_{4}, \quad \mathrm{BaTiO}_{3} /\left(\mathrm{CoFe}_{2} \mathrm{O}_{4} / \mathrm{Ti}_{2} \mathrm{O}_{4}\right)$, and $\mathrm{BaTiO}_{3} /$ $\mathrm{Ni}_{0.97} \mathrm{Co}_{0.03} \mathrm{Mn}_{0.10} \mathrm{Fe}_{1.90} \mathrm{O}_{4}{ }^{7,8}$ Higher coefficients can be achieved by combining materials with larger strictive coefficients such as PMN and Terfenol. ${ }^{9-12}$ The value of $\alpha^{E}$ depends on the respective orientations of the field vectors ${ }^{13}$ and can be increased in resonant composites. ${ }^{14}$

The ferromagnetic components in the above composites possess a significant magnetocrystalline anisotropy. Therefore, a large magnetic bias field is required to develop a sizable ME voltage. This disadvantage can be circumvented by selecting amorphous ferromagnetic components such as amorphous $\mathrm{FeCoSiB}$ (Ref. 15) whose magnetostriction, however, is not large. ${ }^{16} \mathrm{TbFe} / \mathrm{FeCo}$ multilayers are both mag-

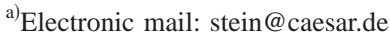

netically soft and highly strictive. ${ }^{17}$ They have thus been deposited onto highly strictive single-crystal PMN-PT substrates to produce candidates for miniaturized magneticfield sensors. The preparation and ME properties of micronsized single-crystal PMN-PT $\| \mathrm{TbFe} / / \mathrm{FeCo}$ multilayers $\mathrm{ME}$ composites are described in this publication.

\section{FABRICATION}

The investigated ME composites consist of a piezoelectric substrate on both sides of which a magnetostrictive multilayer was sputter deposited. During each deposition the edges of the substrate were covered to inhibit short circuiting. The $\mathrm{Tb}_{40} \mathrm{Fe}_{60} / \mathrm{Fe}_{50} \mathrm{Co}_{50}$ multilayers were magnetron sputtered with a Von Ardenne CS 730S tool using cast targets (TbFe: diameter of $10 \mathrm{~cm}$ and FeCo: $20 \mathrm{~cm}$ ). The base and Ar sputtering pressures were kept at $7 \times 10^{-8}$ and 4 $\times 10^{-3}$ mbar, respectively. During the room-temperature deposition of $\mathrm{TbFe}$ and $\mathrm{FeCo}$ layers the rf power was adjusted to 125 and $350 \mathrm{~W}$, resulting in respective deposition rates of 0.55 and $0.22 \mathrm{~nm} / \mathrm{s}$. Both materials were deposited with a magnetic bias field of $10 \mathrm{mT}$ to induce an easy axis in the plane of the multilayer. The resulting multilayer had the composition $[\mathrm{TbFe}(7 \mathrm{~nm}) / \mathrm{FeCo}(10 \mathrm{~nm})]_{262}$ with a total thickness of $4 \mu \mathrm{m}$.

The piezoelectric substrate was a $100-\mu \mathrm{m}$-thick $\langle 001\rangle$ oriented $0.68 \mathrm{~Pb}\left(\mathrm{Mg}_{1 / 3} \mathrm{Nb}_{2 / 3}\right) \mathrm{O}_{3}-0.32 \mathrm{PbTiO}_{3}$ (PMN-PT) piezoelectric single crystal with lateral dimensions of 5 $\times 5 \mathrm{~mm}^{2}$. It was poled perpendicular to the substrate plane.

\section{MEASUREMENT METHOD}

The ME composite was electroded and mounted on a carrier. In order to connect the bottom electrode as unconstrained as possible one side of the ME composite was glued at its center to the tip of a small pyramid formed of conduc- 


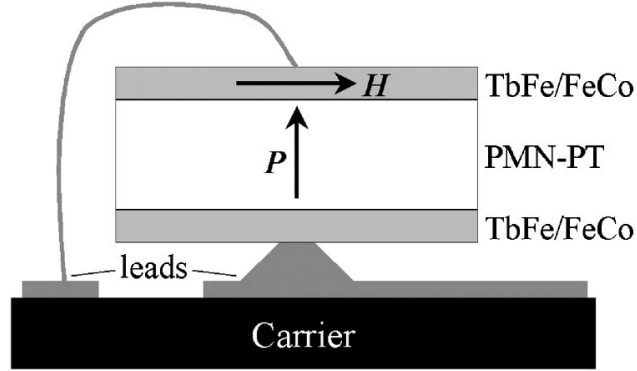

FIG. 1. ME composite layout and measurement setup for longitudinaltransversal (LT) mode alignment.

tive silver sitting on the carrier. A $25-\mu \mathrm{m}$-diameter aluminum wire lead to the top electrode was fabricated with the aid of a wire bonder. The carrier was mounted inside an electromagnet where a dc magnetic field up to $1 \mathrm{~T}$ could be applied. The Helmholtz coils positioned on the pole shoes of the solenoid allowed superposition of the de field, $H$, and an ac field, $H_{\mathrm{ac}}$, of up to $10 \mathrm{Oe}$ (peak). The voltage drop across the sample was recorded via a lock-in amplifier.

During the measurement the ME samples were aligned for the longitudinal-transversal (LT) mode, as shown in Fig. 1.

The voltage that develops at the sample leads consists of a part stemming from the ME effect, $U_{\mathrm{ME}}$, and a part induced by the measuring field $H_{\mathrm{ac}}$ into the loop formed by the sample leads. These contributions differ by a phase angle of $\pi / 2$,

$$
U_{\text {meas }} e^{i(\omega t+\Phi)}=\alpha^{E} H_{\mathrm{ac}} e^{i \omega t}+\gamma H_{\mathrm{ac}} e^{i \omega t+\pi / 2} .
$$

The quantity $\gamma$ indicates the magnitude of the inductively coupled voltage. A phase-sensitive voltage meter was used to separate the ME voltage by adjusting the phase $\Phi$ such that $e^{i \Phi} \rightarrow \pm 1$ as $H \rightarrow \pm \infty$ so that $U_{\mathrm{ME}}=U_{\text {meas }}(0)$.

For magnetization measurements, a Lakeshore vibratingsample magnetometer (VSM) tool was employed.

\section{RESULTS}

Figures 2 and 3 show the magnetization and ME voltages of a $4-\mu \mathrm{m}\left(\mathrm{Tb}_{40} \mathrm{Fe}_{60} / \mathrm{Fe}_{50} \mathrm{Co}_{50}\right)_{262} / / 100-\mu \mathrm{m}$ (PMN$\mathrm{PT}) / / 4-\mu \mathrm{m}\left(\mathrm{Tb}_{40} \mathrm{Fe}_{60} / \mathrm{Fe}_{50} \mathrm{Co}_{50}\right)_{262}$ composite. The graphs identified by the open and closed circles represent measurements in which the applied magnetic bias field was directed parallel to the hard and easy axes, respectively. While the difference in the two resulting $M-H$ characteristics is small

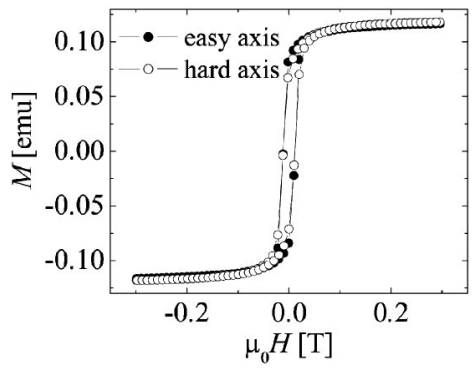

FIG. 2. Room-temperature magnetization of ME sample (4- $\mu \mathrm{m}$ multilayer electrodes) along easy and hard axes measured by vibrating-sample magnetometry (VSM).

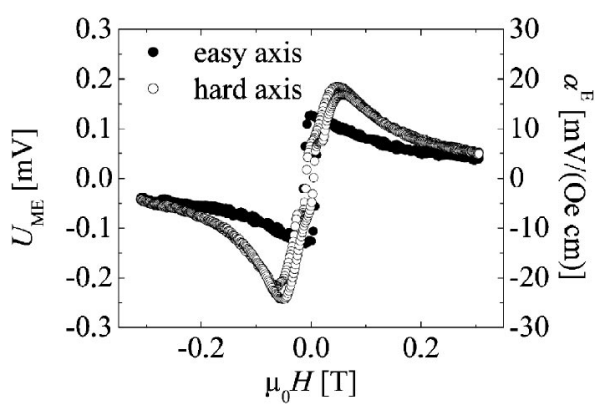

FIG. 3. Dependence of ME voltage on dc magnetic bias field in LT mode, measured with ac field of $f=1 \mathrm{kHz}$ and amplitude of 1 Oe.

(see Fig. 2); the two ME voltage-bias field characteristics differ significantly (see Fig. 3). Since in the LT mode the latter represents in essence the derivative $\partial \lambda / \partial H \propto \partial M / \partial H$ [see Eq. (3)] the enhancement of the difference is readily understood. Both characteristics in Fig. 3 exhibit the small hysteresis characteristic of a soft magnetic material.

In hard axis alignment, the maximum $\alpha^{E}$ reaches a value of $19 \mathrm{mV} /(\mathrm{Oe} \mathrm{cm})$ at a bias field of $40 \mathrm{mT}$. Although there is no asymmetry in peak position, the highest value in the opposite sweep direction at $-40 \mathrm{mT}$ lies at $-24 \mathrm{mV} /(\mathrm{Oe} \mathrm{cm})$. The easy axis measurement shows no asymmetry and the maximum ME coefficient is $13 \mathrm{mV} /(\mathrm{Oe} \mathrm{cm})$ at a bias field of $2 \mathrm{mT}$. This asymmetry can be attributed to anisotropies: either the deposition area is asymmetric due to the covering of the edges or both magnetic electrodes are slightly misaligned regarding the sputtering bias field.

Figure 4 shows the linear dependence of the ME voltage drop on the applied ac field amplitude.

\section{DISCUSSION}

The ME voltage characteristic $U_{\mathrm{ME}}=f\left(\mu_{0} H\right)$ shown in Fig. 3 demonstrates that the magnitude of the ME voltage depends on the orientation of the easy axis of the $\mathrm{TbFe} / \mathrm{FeCo}$ multilayer and that its maximum occurs at a small bias field of $H=2$ and $40 \mathrm{mT}$, respectively. The influence of the orientation of the easy axis with respect to the probing field $H_{\mathrm{ac}}$ can be understood by observing that magnetic switching between easy axes involves fewer $90^{\circ}$ domain boundaries than switching between hard axes. The small magnitude of the bias field maximizing the ME voltage, $H_{\text {bias, }}$, is a consequence of the small anisotropy field of the $\mathrm{TbFe} / \mathrm{FeCo}$ multilayers, as can be seen from expressing Eq. (1b) as

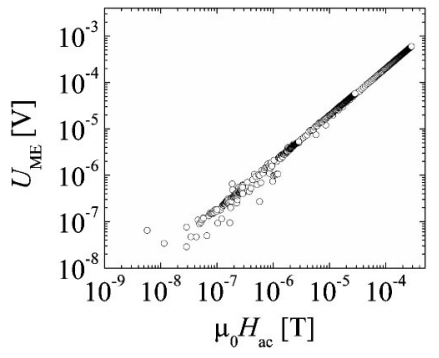

FIG. 4. Dependence of ME voltage on ac magnetic field (peak value). Hard axis alignment measured with ac frequency of $1 \mathrm{kHz}$ at dc field of $60 \mathrm{mT}$. 


$$
\alpha^{E}=\left.\frac{\partial \lambda}{\partial H}\right|_{H_{\text {bias }}} \frac{\partial E}{\partial \lambda},
$$

where the quantity $\lambda$ represents the magnetostriction of the multilayer.

\section{CONCLUSION}

In a step towards miniaturized high-performance ME sensors a composite consisting of $100-\mu \mathrm{m}$ ferroelectric PMN-PT with double-sided 4- $\mu \mathrm{m}$ magnetostrictive magnetically soft $\left(\mathrm{Tb}_{40} \mathrm{Fe}_{60} / \mathrm{Fe}_{50} \mathrm{Co}_{50}\right)_{262}$ "electrodes" was fabricated. Due to the excessive sputtering times required to deposit electrodes with a thickness comparable to that of the PMN substrate, only elastically mismatched composites with small ME coefficients were manufactured. It was observed, though, that the ME voltage is proportional to the thickness of the electrodes, as expected, if their thickness is small in comparison to that of the PMN-PT single-crystal substrate. If the observed ME voltage is converted to what would be observed in elastically matched composites the present values are comparable to those observed in bulk composites.

\section{ACKNOWLEDGMENTS}

This work is supported by the Office of Naval Research Grant No. MURI N000140110761, by the ONR London of- fice Grant No. NICOP N000140210231, and by the National Science Foundation, Grant No. DMR0095166.

${ }^{1}$ G. A. Smolenskii, V. M. Yudin, E. S. Sher, and Y. E. Stolypin, Sov. Phys. JETP 16, 622 (1963).

${ }^{2}$ Y. E. Roginskaya, Y. Y. Tomashpol'skii, Y. N. Venevtsev, V. M. Petrov, and G. S. Zhdanov, Sov. Phys. JETP 23, 47 (1966).

${ }^{3}$ I. Sosnowska, T. Peterlin-Neumaier, and E. Steichele, J. Phys. C 15, 4835 (1982).

${ }^{4}$ J. Wang et al., Science 299, 1719 (2003).

${ }^{5}$ B. B. Van Aken, T. T. M. Palstra, A. Filippetti, and N. A. Spaldin, Nat. Mater. 3, 164 (2004).

${ }^{6}$ J. V. Suchtelen, Philips Res. Rep. 27, 28 (1972).

${ }^{7}$ J. V. D. Boomgaard, A. M. Run, and J. V. Suchtelen, Ferroelectrics 10, 295 (1976).

${ }^{8}$ J. V. D. Boomgaard, A. M. Run, and J. V. Suchtelen, Ferroelectrics 14, 727 (1976).

${ }^{9}$ J. Ryu, S. Priya, K. Uchino, and H. E. Kim, J. Electroceram. 8, 107 (2002)

${ }^{10} \mathrm{~S}$. X. Dong, J. F. Li, and D. Viehland, IEEE Trans. Ultrason. Ferroelectr. Freq. Control 50, 1236 (2003).

${ }^{11}$ U. Laletsin, N. Padubnaya, G. Srinivasan, and C. P. Devreugd, Appl. Phys. A: Mater. Sci. Process. 78, 33 (2004).

${ }^{12}$ G. Srinivasan, E. T. Rasmussen, J. Gallegos, R. Srinivasan, Y. I. Bokhan, and V. M. Laletin, Phys. Rev. B 64, 214408 (2001).

${ }^{13}$ S. Dong, J. F. Li, and D. Viehland, J. Appl. Phys. 95, 2625 (2004).

${ }^{14}$ S. X. Dong, J. R. Cheng, J. F. Li, and D. Viehland, Appl. Phys. Lett. 83, 4812 (2003).

${ }^{15}$ K. H. Shin, M. Inoue, and K. I. Arai, IEEE Trans. Magn. 34, 1324 (1998).

${ }^{16}$ J. D. Livingston, Phys. Status Solidi A 70, 591 (1982).

${ }^{17}$ E. Quandt and A. Ludwig, J. Appl. Phys. 85, 6232 (1999). 